\title{
Variational Iteration Method for a Fractional-Order Brusselator System
}

\author{
H. Jafari, ${ }^{1}$ Abdelouahab Kadem, ${ }^{2}$ and D. Baleanu ${ }^{3,4,5}$ \\ ${ }^{1}$ Department of Mathematics (Pure and Applied), Rhodes University, P.O. Box 946140, Grahamstown, South Africa \\ ${ }^{2}$ LMFN Mathematics Department, University of Setif, 19000, Algeria \\ ${ }^{3}$ Department of Chemical and Materials Engineering, King Abdulaziz University, P.O. Box 80204, Jeddah 21589, Saudi Arabia \\ ${ }^{4}$ Department of Mathematics and Computer Sciences, Cankaya University, Balgat 06530, Ankara, Turkey \\ ${ }^{5}$ Institute of Space Sciences, P.O. BOX MG-23, 76900 Magurele, Bucharest, Romania
}

Correspondence should be addressed to H. Jafari; jafari@umz.ac.ir

Received 13 December 2013; Accepted 10 February 2014; Published 18 March 2014

Academic Editor: Alexander Domoshnitsky

Copyright (c) $2014 \mathrm{H}$. Jafari et al. This is an open access article distributed under the Creative Commons Attribution License, which permits unrestricted use, distribution, and reproduction in any medium, provided the original work is properly cited.

This paper presents approximate analytical solutions for the fractional-order Brusselator system using the variational iteration method. The fractional derivatives are described in the Caputo sense. This method is based on the incorporation of the correction functional for the equation. Two examples are solved as illustrations, using symbolic computation. The numerical results show that the introduced approach is a promising tool for solving system of linear and nonlinear fractional differential equations.

\section{Introduction}

In recent years, fractional differential equations (FDEs) have been the focus of many studies due to their appearance in various fields such as physics, chemistry, and engineering [1-3]. On the other hand, much attention has been paid to the solutions of fractional differential equations. Since most fractional differential equations do not have exact analytic solutions and approximate and numerical techniques, therefore, they are used extensively. Recently, the Adomian decomposition method, homotopy perturbation method, homotopy analysis method, and differential transform method have been used for solving a wide range of problems [4-10].

Another powerful analytical method, called the variational iteration method (VIM), was first introduced in [11]. This technique has successfully been applied to many situations: for example, see [12-17]. Reference [18] was the first where the variational iteration method was applied to fractional differential equations. Odibat and Momani [19] implemented the variational iteration method to solve partial differential equations of fractional order.

In this paper, we introduce a new application of the variational iteration method to provide approximate solutions of the fractional-order Brusselator system in the following form:

$$
\begin{aligned}
& D_{t}^{\alpha_{1}} x(t)=a-(\mu+1) x(t)+x(t)^{2} y(t), \\
& D_{t}^{\alpha_{2}} y(t)=\mu x(t)-x(t)^{2} y(t),
\end{aligned}
$$

subject to the initial conditions

$$
x(0)=c_{1}, \quad y(0)=c_{2},
$$

with $a>0, \mu>0,0<\alpha_{i} \leq 1(i=1,2)$, and $c_{1}, c_{2}$ are constants.

$D^{\alpha_{i}}$ is used to represent the Caputo-type fractional derivative of order $\alpha_{i}$.

The Riemann-Liouville definition of the fractional integration [2] is given by

$$
I_{t}^{\alpha} x(t)=\frac{1}{\Gamma(\alpha)} \int_{0}^{t}(t-\zeta)^{\alpha-1} x(\zeta) d \zeta \quad \alpha>0, t>0
$$


For our purpose in this paper, we adopt Caputo's fractional derivative [2]:

$$
\begin{aligned}
& D_{t}^{\alpha} x(t) \\
& \quad= \begin{cases}\frac{1}{\Gamma(n-\alpha)} \int_{0}^{t}(t-\zeta)^{n-\alpha-1} x^{(n)}(\zeta) d \zeta & n-1<\alpha<n, \\
\frac{d^{n} x(t)}{d t^{n}} & \alpha=n,\end{cases}
\end{aligned}
$$

where $n$ is a positive integer and $\Gamma(\cdot)$ is the Gamma function. In particular, $0<\alpha_{i}<1$, and we have

$$
D_{t}^{\alpha} x(t)=\frac{1}{\Gamma(1-\alpha)} \int_{0}^{t}(t-\zeta)^{-\alpha} x^{\prime}(\zeta) d \zeta
$$

The fractional-order Brusselator system has been considered by several authors recently [20-22]. Gafiychuk and Datsko investigated its stability [20]. Wang and Li proved by numerical method that the solutions of the fractionalorder Brusselator system have a limit cycle [22]. We used the variational iteration method to investigate the approximate solutions of the fractional-order Brusselator system.

\section{Variational Iteration Method}

The principles of the variational iteration method and its applicability for various kinds of differential equations are given in $[23,24]$. In [18], it was shown that the variational iteration method is also valid for fractional differential equations. In this section, following the discussion presented in [18], we extend the application of the variational iteration method to solve the fractional Brusselator equation:

$$
\begin{aligned}
& D_{t}^{\alpha_{1}} x(t)=a-(\mu+1) x(t)+x(t)^{2} y(t), \\
& D_{t}^{\alpha_{2}} y(t)=\mu x(t)-x(t)^{2} y(t) .
\end{aligned}
$$

According to the variational iteration method, we can construct the correction functional for (6) as

$$
\begin{aligned}
x_{n+1}(t)=x_{n}(t)+I_{t}^{\alpha_{1}}[ & \lambda_{1}\left(D_{t}^{\alpha_{1}} x_{n}(t)-a+(\mu+1) x_{n}(t)\right. \\
& \left.\left.-x_{n}^{2}(t) y_{n}(t)\right)\right] \\
= & x_{n}(t)+\frac{1}{\Gamma\left(\alpha_{1}\right)} \int_{0}^{t}(t-\zeta)^{\alpha_{1}-1} \lambda_{1}(\zeta) \\
& \times\left(D_{\zeta}^{\alpha_{1}} x_{n}(\zeta)-a+(\mu+1) x_{n}(\zeta)\right. \\
& \left.-x_{n}^{2}(\zeta) y_{n}(\zeta)\right) d \zeta
\end{aligned}
$$

$$
\begin{aligned}
y_{n+1}(t)=y_{n}(t)+I_{t}^{\alpha_{2}}[ & \lambda_{2}\left(D_{t}^{\alpha_{2}} y_{n}(t)-\mu x_{n}(t)\right. \\
& \left.\left.+x_{n}^{2}(t) y_{n}(t)\right)\right] \\
= & y_{n}(t)+\frac{1}{\Gamma\left(\alpha_{2}\right)} \int_{0}^{t}(t-\zeta)^{\alpha_{2}-1} \lambda_{2}(\zeta) \\
& \times\left(D_{\zeta}^{\alpha_{2}} y_{n}(\zeta)-\mu x_{n}(\zeta)+x_{n}^{2}(\zeta) y_{n}(\zeta)\right) d \zeta
\end{aligned}
$$

where $\lambda_{i},(i=1,2)$ are the general Lagrange multiplier, which can be identified optimally via variational theory $[25,26]$.

To identify approximately Lagrange multiplier, some approximations must be made. The correction functional equation (7) can be approximately expressed as follows:

$$
\begin{array}{r}
x_{n+1}(t)=x_{n}(t)+\int_{0}^{t} \lambda_{1}(\zeta)\left(x_{n}^{\prime}(\zeta)-a+(\mu+1) \tilde{x}_{n}(\zeta)\right. \\
\left.-\widetilde{x}_{n}^{2}(\zeta) \tilde{y}_{n}(\zeta)\right) d \zeta \\
y_{n+1}(t)=y_{n}(t)+\int_{0}^{t} \lambda_{2}(\zeta)\left(y_{n}^{\prime}(\zeta)-\mu \tilde{x}_{n}(\zeta)\right. \\
\left.+\tilde{x}_{n}^{2}(\zeta) \tilde{y}_{n}(\zeta)\right) d \zeta
\end{array}
$$

where $\tilde{x}_{n}$ and $\tilde{y}_{n}$ are considered as restricted variations, in which $\delta \widetilde{x}_{n}=\delta \widetilde{y}_{n}=0$. To find the optimal $\lambda_{1}$ and $\lambda_{2}$, we proceed as follows:

$$
\begin{array}{r}
\delta x_{n+1}(t)=\delta x_{n}(t)+\delta \int_{0}^{t} \lambda_{1}(\zeta)\left(x_{n}^{\prime}(\zeta)-a+(\mu+1) \tilde{x}_{n}(\zeta)\right. \\
\left.-\tilde{x}_{n}^{2}(\zeta) \tilde{y}_{n}(\zeta)\right) d \zeta=0 \\
\delta y_{n+1}(t)=\delta y_{n}(t)+\delta \int_{0}^{t} \lambda_{2}(\zeta)\left(y_{n}^{\prime}(\zeta)-\mu \tilde{x}_{n}(\zeta)\right. \\
\left.+\tilde{x}_{n}^{2}(\zeta) \tilde{y}_{n}(\zeta)\right) d \zeta=0
\end{array}
$$

The stationary conditions can be obtained as follows:

$$
\begin{array}{ll}
\left.\lambda_{1}^{\prime}(\zeta)\right|_{\zeta=t}=0, & 1+\left.\lambda_{1}(\zeta)\right|_{\zeta=t}=0 \Longrightarrow \lambda_{1}(\zeta)=-1, \\
\left.\lambda_{2}^{\prime}(\zeta)\right|_{\zeta=t}=0, & 1+\left.\lambda_{2}(\zeta)\right|_{\zeta=t}=0 \Longrightarrow \lambda_{2}(\zeta)=-1 .
\end{array}
$$

We substitute $\lambda_{i}(\zeta)=-1,(i=1,2)$ into the functional equation (11) to obtain the following iteration formula:

$$
\begin{aligned}
x_{n+1}(t)=x_{n}(t)-I_{t}^{\alpha_{1}}[ & D_{t}^{\alpha_{1}} x_{n}(t)-a+(\mu+1) x_{n}(t) \\
& \left.-x_{n}^{2}(t) y_{n}(t)\right], \\
y_{n+1}(t)=y_{n}(t)-I_{t}^{\alpha_{2}}[ & D_{t}^{\alpha_{2}} y_{n}(t)-\mu x_{n}(t) \\
+ & \left.x_{n}^{2}(t) y_{n}(t)\right] .
\end{aligned}
$$




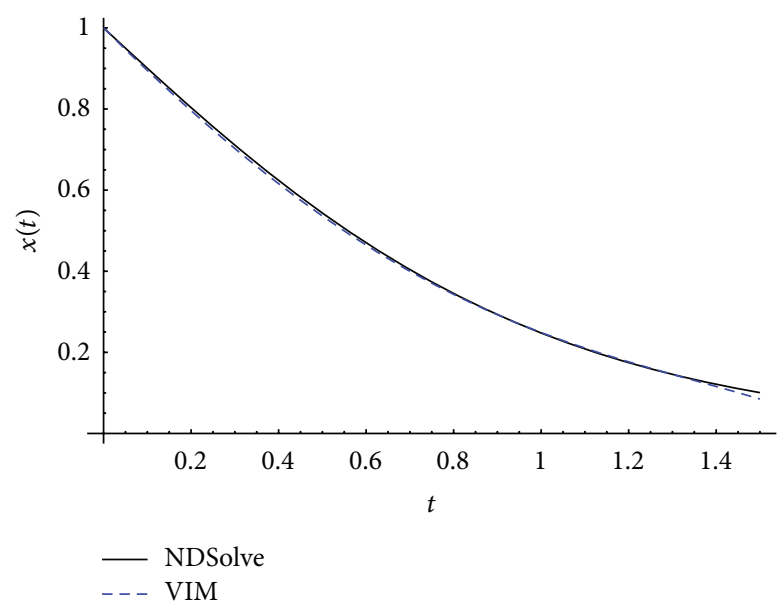

(a)

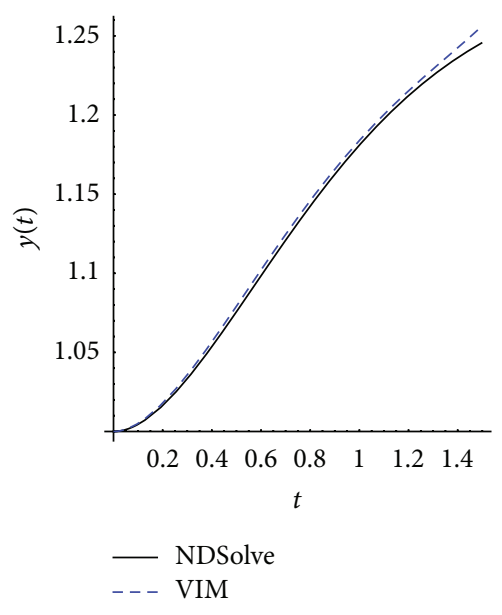

(b)

Figure 1

The initial approximations $x_{0}(t)$ and $y_{0}(t)$ can be freely chosen if they satisfy the initial conditions of the problem. Finally, we approximate the solutions $x(t)=\lim _{n \rightarrow 1} x_{n}(t)$ and $y(t)=\lim _{n \rightarrow 1} y_{n}(t)$ by the $n$th terms $x_{n}(t)$ and $y_{n}(t)$.

\section{Illustrative Examples}

For purposes of illustration of (VIM) for solving Brusselator equation, we present two examples.

Example 1. Consider the following fractional-order Brusselator system:

$$
\begin{aligned}
& D_{t}^{\alpha_{1}} x(t)=-2 x(t)+x(t)^{2} y(t), \\
& D_{t}^{\alpha_{2}} y(t)=x(t)-x(t)^{2} y(t),
\end{aligned}
$$

with the initial conditions:

$$
x(0)=1, \quad y(0)=1 .
$$

According to the variational iteration method and (11), the iteration formula for (12) is given by

$$
\begin{array}{r}
x_{n+1}(t)=x_{n}(t)-I_{t}^{\alpha_{1}}\left[D_{t}^{\alpha_{1}} x_{n}(t)+2 x_{n}(t)\right. \\
\left.-x_{n}^{2}(t) y_{n}(t)\right], \\
y_{n+1}(t)=y_{n}(t)-I_{t}^{\alpha_{2}}\left[D_{t}^{\alpha_{2}} y_{n}(t)-x_{n}(t)\right. \\
\left.+x_{n}^{2}(t) y_{n}(t)\right] .
\end{array}
$$

By using the above variational iteration formula, if we start with the initial approximations $x_{0}(t)=1$ and $y_{0}(t)=1$, we can obtain directly the other components as

$$
\begin{aligned}
& x_{1}(t)=1-\frac{t^{\alpha_{1}}}{\Gamma\left[\alpha_{1}+1\right]}, \\
& y_{1}(t)=1, \\
& x_{2}(t)=1-\frac{t^{\alpha_{1}}}{\Gamma\left[\alpha_{1}+1\right]}+\frac{t^{3 \alpha_{1}} \Gamma\left[2 \alpha_{1}+1\right]}{\Gamma\left[1+\alpha_{1}\right]^{2} \Gamma\left[1+3 \alpha_{1}\right]}, \\
& y_{2}(t)=1+\frac{t^{\alpha_{1}+\alpha_{2}}}{\Gamma\left[\alpha_{1}+\alpha_{2}+1\right]}-\frac{t^{2 \alpha_{1}+\alpha_{2}} \Gamma\left[2 \alpha_{1}+1\right]}{\Gamma\left[\alpha_{1}+1\right]^{2} \Gamma\left[1+2 \alpha_{1}+\alpha_{2}\right]},
\end{aligned}
$$

and so on; in the same way the rest of the components of the iteration formula can be obtained. Figures $1(a)$ and $1(b)$ show comparison between the approximate solutions $(x(t) \cong$ $\left.x_{5}(t)\right),\left(\left(y(t) \cong y_{5}(t)\right)\right.$ of (12) obtained using VIM for the special case $\alpha_{1}=\alpha_{2}=0.98$ and the numerical solutions for the special case $\alpha_{1}=\alpha_{2}=1$, respectively. Figures $2(\mathrm{a})$ and 2(b) show the approximate solutions $\left(x(t) \cong x_{5}(t)\right),((y(t) \cong$ $\left.y_{5}(t)\right)$ of (12) using VIM for the special case $\alpha_{1}=\alpha_{2}=1$ and the numerical solutions, respectively.

Example 2. Consider the following fractional-order Brusselator system:

$$
\begin{aligned}
& D^{\alpha_{1}} x(t)=0.5-1.1 x(t)+x(t)^{2} y(t), \\
& D^{\alpha_{2}} y(t)=0.1 x(t)-x(t)^{2} y(t),
\end{aligned}
$$




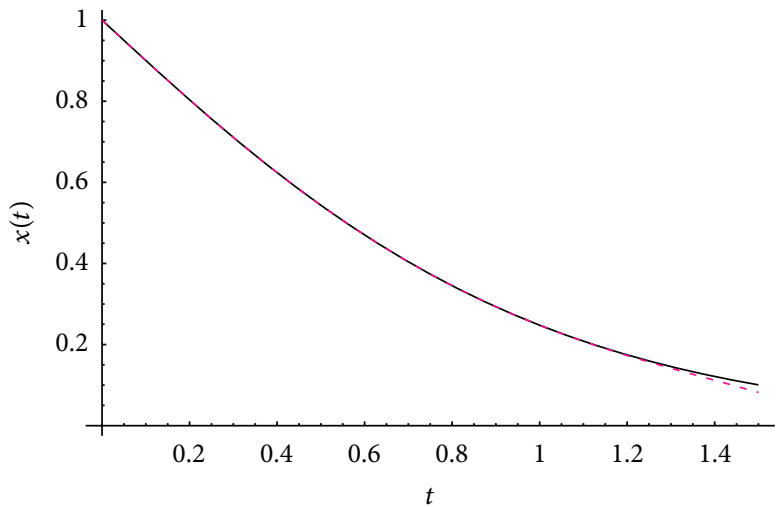

- NDSolve - - VIM

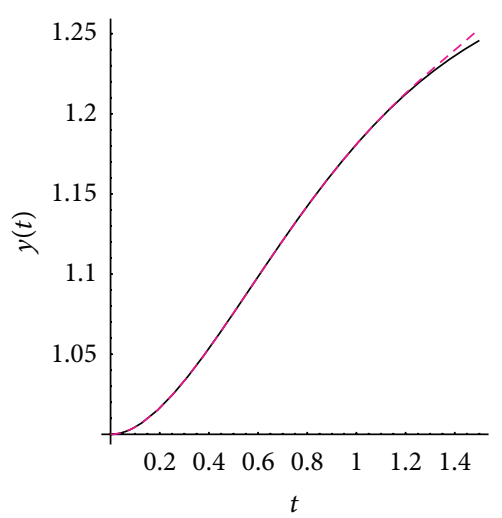

- NDSolve

(a)

(b)

Figure 2

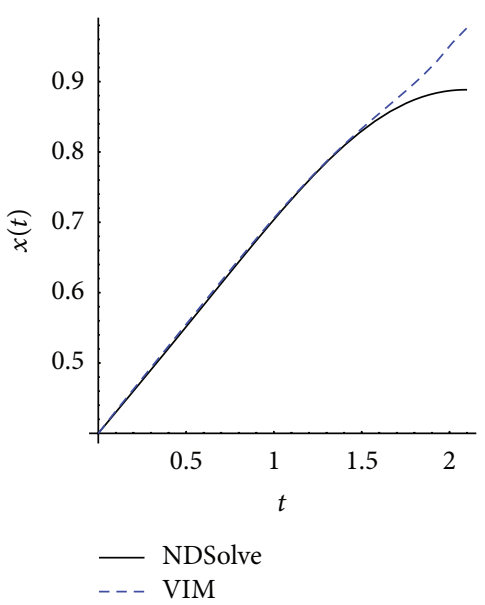

(a)

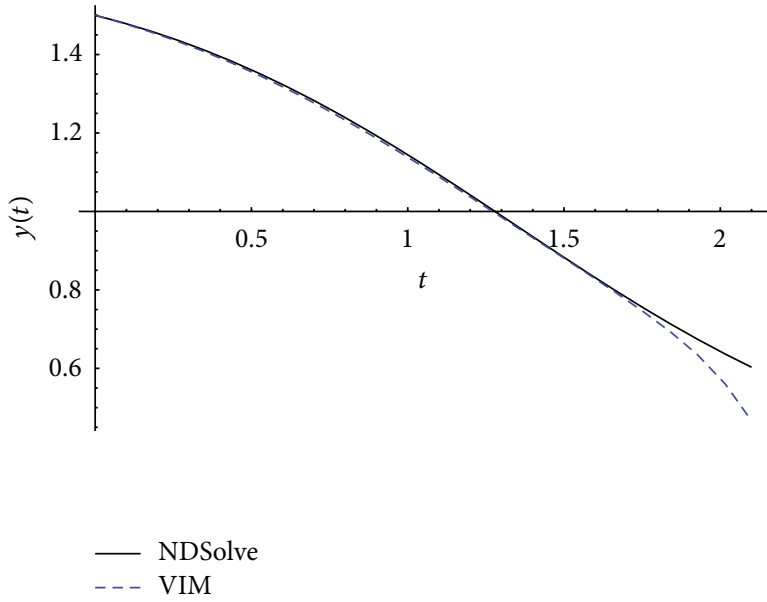

(b)

Figure 3

with the initial conditions:

$$
x(0)=0.4, \quad y(0)=1.5 \text {. }
$$

The correction functional for (16) turns out to be

$$
\begin{aligned}
x_{n+1}(t)=x_{n}(t)-I_{t}^{\alpha_{1}} & {\left[D_{t}^{\alpha_{1}} x_{n}(t)-0.5+1.1 x_{n}(t)\right.} \\
& \left.-x_{n}^{2}(t) y_{n}(t)\right], \\
y_{n+1}(t)=y_{n}(t)-I_{t}^{\alpha_{2}} & {\left[D_{t}^{\alpha_{2}} y_{n}(t)-0.1 x_{n}(t)\right.} \\
& \left.+x_{n}^{2}(t) y_{n}(t)\right] .
\end{aligned}
$$

By the above variational iteration formula and beginning with the initial approximations $x_{0}(t)=0.4+\left(0.5 \times t^{\alpha_{1}} / \Gamma\left[\alpha_{1}+1\right]\right)$ and $y_{0}(t)=1.5$, we can obtain directly the other components as

$$
\begin{aligned}
x_{1}(t)= & 0.4+\frac{0.5 t^{\alpha_{1}}}{\Gamma\left[\alpha_{1}+1\right]}+\frac{0.05 t^{2 \alpha_{1}}}{\Gamma\left[2 \alpha_{1}+1\right]} \\
& +\frac{0.119316^{\alpha_{1}} t^{3 \alpha_{1}}}{\Gamma\left[2 \alpha_{1}+1\right] \Gamma\left[3 \alpha_{1}+1\right]}-\frac{0.2 t^{\alpha_{1}}}{\Gamma\left[\alpha_{1}+1\right]}, \\
y_{1}(t)= & 1.5-\frac{0.55 t^{\alpha_{1}+\alpha_{2}}}{\Gamma\left[\alpha_{1}+\alpha_{2}+1\right]} \\
& -\frac{0.375 t^{2 \alpha_{1}+\alpha_{2}} \Gamma\left[2 \alpha_{1}+1\right]}{\Gamma\left[\alpha_{1}+1\right]^{2} \Gamma\left[2 \alpha_{1}+\alpha_{2}+1\right]}-\frac{0.2 t^{\alpha_{2}}}{\Gamma\left[\alpha_{2}+1\right]},
\end{aligned}
$$

and so on; in the same manner the remaining set of the components of the iteration formula can be obtained. Figures 3(a) 


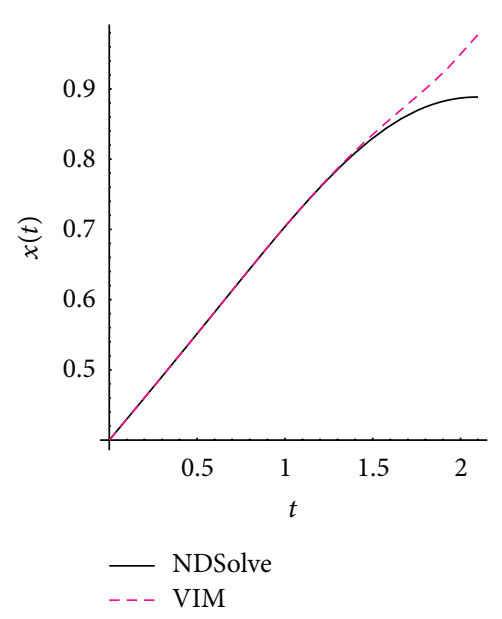

(a)

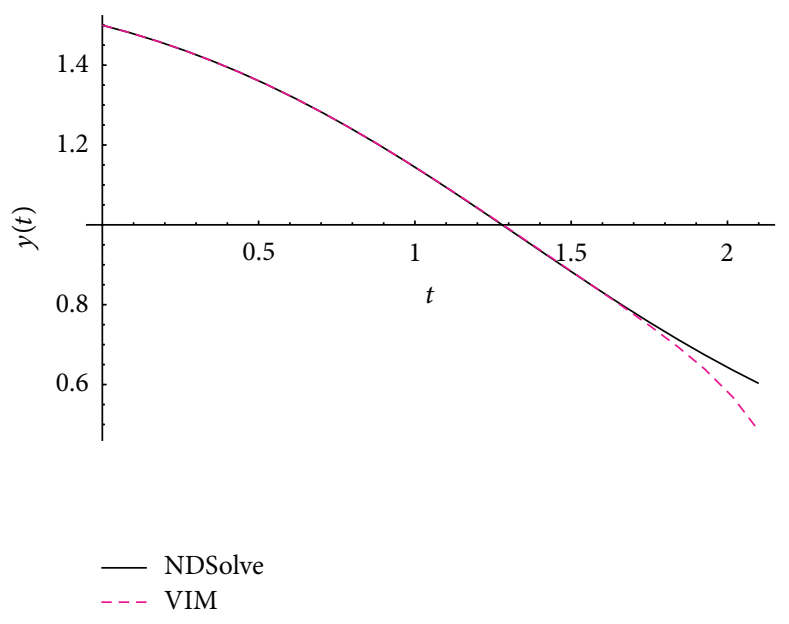

(b)

FIGURE 4

and 3(b) show comparison between the approximate solutions $\left(x(t) \cong x_{4}(t)\right),\left(\left(y(t) \cong y_{4}(t)\right)\right.$ of $(16)$ obtained using VIM for the special case $\alpha_{1}=\alpha_{2}=.98$ and the numerical solutions for the special case $\alpha_{1}=\alpha_{2}=1$, respectively. Figures $4(\mathrm{a})$ and $4(\mathrm{~b})$ show the approximate solutions $(x(t) \cong$ $\left.x_{4}(t)\right),\left(\left(y(t) \cong y_{4}(t)\right)\right.$ of (16) using VIM for the special case $\alpha_{1}=\alpha_{2}=1$ and the numerical solutions, respectively.

\section{Conclusions}

The variational iteration method is a powerful method which is able to handle linear/nonlinear fractional differential equations. The method has been applied to fractional-order Brusselator system in order to find its approximate solutions. The results show that the applied method is suitable and inexpensive for obtaining the approximate solutions.

\section{Conflict of Interests}

The authors declare that there is no conflict of interests regarding the publication of this paper.

\section{References}

[1] R. Hilfer, Applications of Fractional Calculus in Physics, Academic Press, Orlando, Fla, USA, 1999.

[2] I. Podlubny, Fractional Differential Equations, Academic Press, San Diego, Calif, USA, 1999.

[3] A. M. Spasic and M. P. Lazarevic, "Electroviscoelasticity of liquid/liquid interfaces: fractional-order model," Journal of Colloid and Interface Science, vol. 282, no. 1, pp. 223-230, 2005.

[4] V. Erturk, S. Momani, and Z. Odibat, "Application of generalized differential transform method to multi-order fractional differential equations," Communications in Nonlinear Science and Numerical Simulation, vol. 13, no. 8, pp. 1642-1654, 2008.

[5] H. Jafari and V. Daftardar-Gejji, "Solving linear and nonlinear fractional diffusion and wave equations by Adomian decomposition," Applied Mathematics and Computation, vol. 180, no. 2, pp. $488-497,2006$.
[6] H. Jafari and S. Momani, "Solving fractional diffusion and wave equations by modified homotopy perturbation method," Physics Letters A, vol. 370, no. 5-6, pp. 388-396, 2007.

[7] H. Jafari and S. Seifi, "Solving a system of nonlinear fractional partial differential equations using homotopy analysis method," Communications in Nonlinear Science and Numerical Simulation, vol. 14, no. 5, pp. 1962-1969, 2009.

[8] H. Jafari and S. Seifi, "Homotopy analysis method for solving linear and nonlinear fractional diffusion-wave equation," Communications in Nonlinear Science and Numerical Simulation, vol. 14, no. 5, pp. 2006-2012, 2009.

[9] H. Jafari, N. Kadkhoda, H. Tajadodi, and S. A. Hosseini Matikolai, "Homotopy perturbation pade technique for solving fractional Riccati differential equations," International Journal of Nonlinear Sciences and Numerical Simulation, vol. 11, pp. 271275, 2010.

[10] H. Jafari and H. Tajadodi, "He's variational iteration method for solving fractional Riccati differential equation," International Journal of Differential Equations, vol. 2010, Article ID 764738, 8 pages, 2010.

[11] J. H. He, "A new approach to nonlinear partial differential equations," Communications in Nonlinear Science and Numerical Simulation, vol. 2, no. 4, pp. 230-235, 1997.

[12] M. A. Abdou and A. A. Soliman, "New applications of variational iteration method," Physica D, vol. 211, no. 1-2, pp. 1-8, 2005.

[13] J. H. He, "Variational iteration method for autonomous ordinary differential systems," Applied Mathematics and Computation, vol. 114, no. 2-3, pp. 115-123, 2000.

[14] J. H. He, "Variational principles for some nonlinear partial differential equations with variable coefficients," Chaos, Solitons and Fractals, vol. 19, no. 4, pp. 847-851, 2004.

[15] H. Jafari, Ch. Chun, and C. M. Khalique, "The variational iterationmethod for finding exact solution of nonlinear gas dynamics equations," Zeitschrift fur Naturforschung, vol. 66, no. 3-4, pp. 161-164, 2011.

[16] Y. Khan, N. Faraz, A. Yildirim, and Q. Wu, "Fractional variational iteration method for fractional initial-boundary value problems arising in the application of nonlinear science," 
Computers and Mathematics with Applications, vol. 62, no. 5, pp. 2273-2278, 2011.

[17] X. J. Yang and D. Baleanu, "Fractal heat conduction problem solved by local fractional variation iteration method," Thermal Science, vol. 17, no. 2, pp. 625-628, 2013.

[18] J. H. He, "Approximate analytical solution for seepage flow with fractional derivatives in porous media," Computer Methods in Applied Mechanics and Engineering, vol. 167, no. 1-2, pp. 57-68, 1998.

[19] Z. Odibat and S. Momani, "The variational iteration method: an efficient scheme for handling fractional partial differential equations in fluid mechanics," Computers and Mathematics with Applications, vol. 58, no. 11-12, pp. 2199-2208, 2009.

[20] V. Gafiychuk and B. Datsko, "Stability analysis and limit cycle in fractional system with Brusselator nonlinearities," Physics Letters A, vol. 372, no. 29, pp. 4902-4904, 2008.

[21] S. Kumar, Y. Khan, and A. Yildirim, "A mathematical modeling arising in the chemical systems and its approximate numerical solution," Asia-Pacific Journal of Chemical Engineering, vol. 7, no. 6, pp. 835-840, 2012.

[22] Y. Wang and C. Li, "Does the fractional Brusselator with efficient dimension less than 1 have a limit cycle?" Physics Letters A, vol. 363, no. 5-6, pp. 414-419, 2007.

[23] J. H. He, "Variational iteration method for delay differential equations," Communications in Nonlinear Science and Numerical Simulation, vol. 2, no. 4, pp. 235-236, 1997.

[24] S. Momani and S. Abuasad, "Application of He's variational iteration method to Helmholtz equation," Chaos, Solitons and Fractals, vol. 27, no. 5, pp. 1119-1123, 2006.

[25] M. Inokuti, H. Sekine, and T. Mura, "General use of the Lagrange multiplier in non-linear mathematical physics," in Variational Method in the Mechanics of Solids, S. Nemat-Nasser, Ed., pp. 156-162, Pergamon Press, Oxford, UK, 1978.

[26] H. Jafari and A. Alipoor, "A new method for calculating general lagrange multiplier in the variational iteration method," Numerical Methods for Partial Differential Equations, vol. 27, no. 4, pp. 996-1001, 2011. 


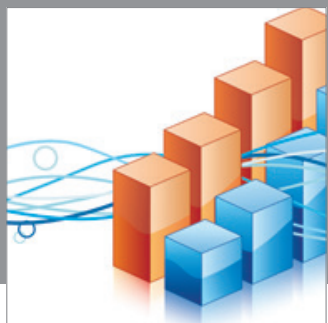

Advances in

Operations Research

mansans

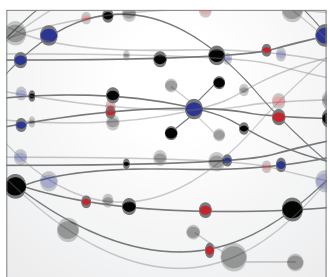

The Scientific World Journal
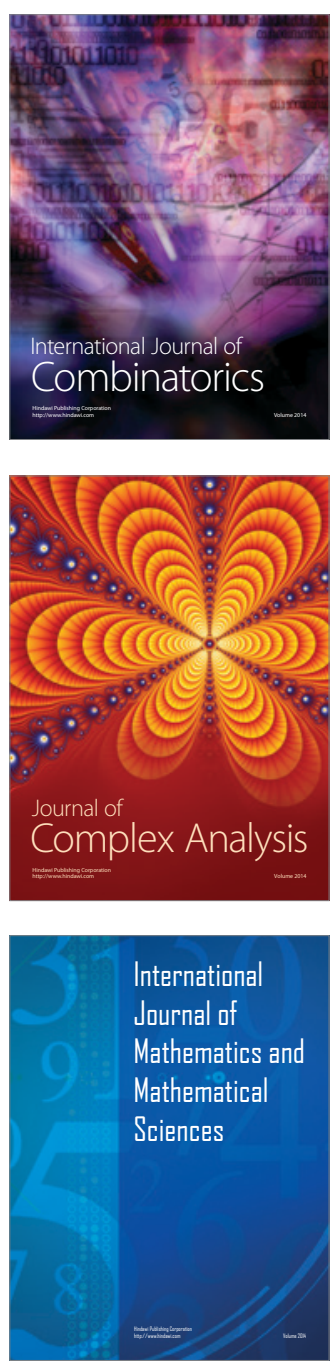
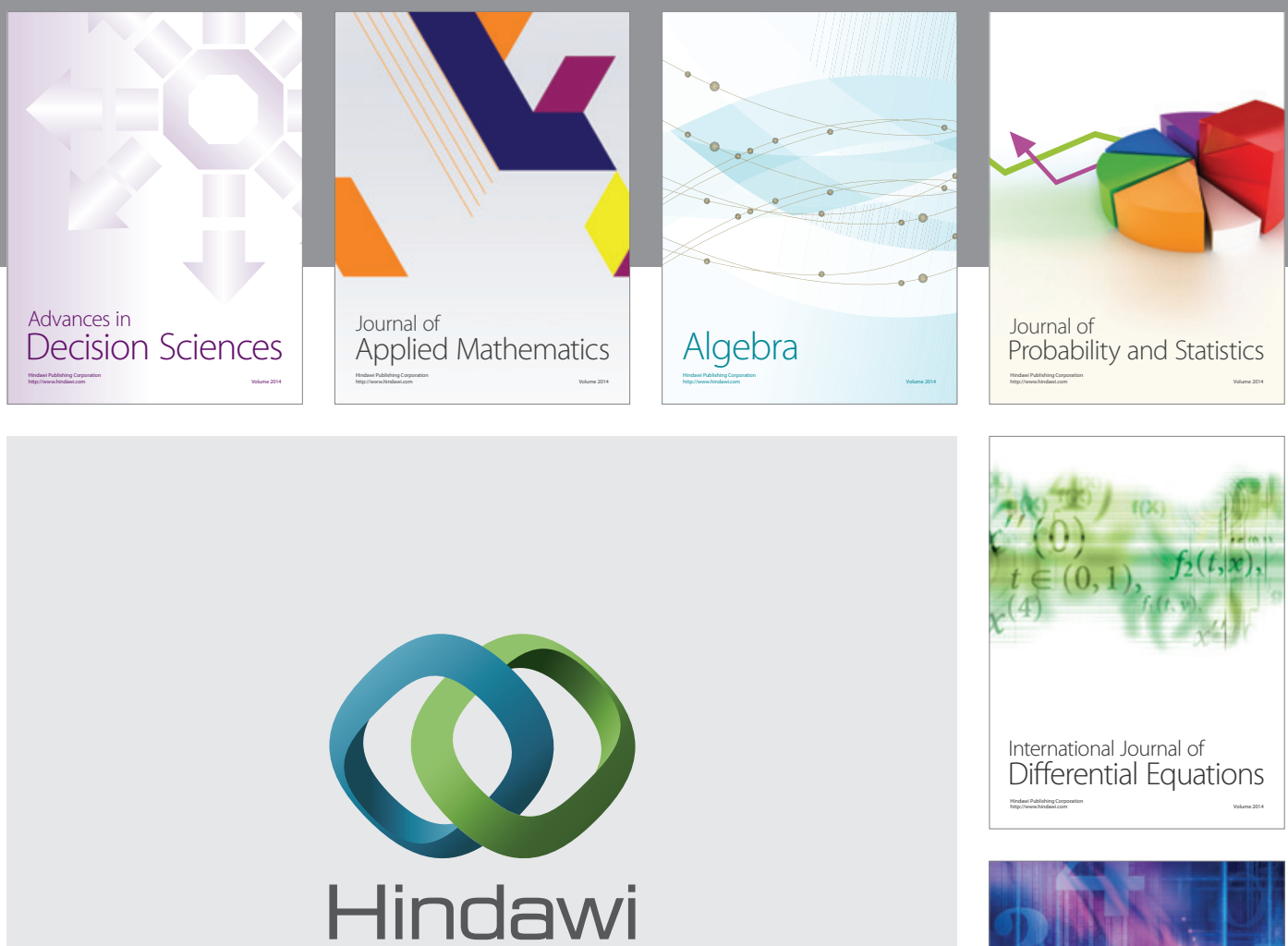

Submit your manuscripts at http://www.hindawi.com
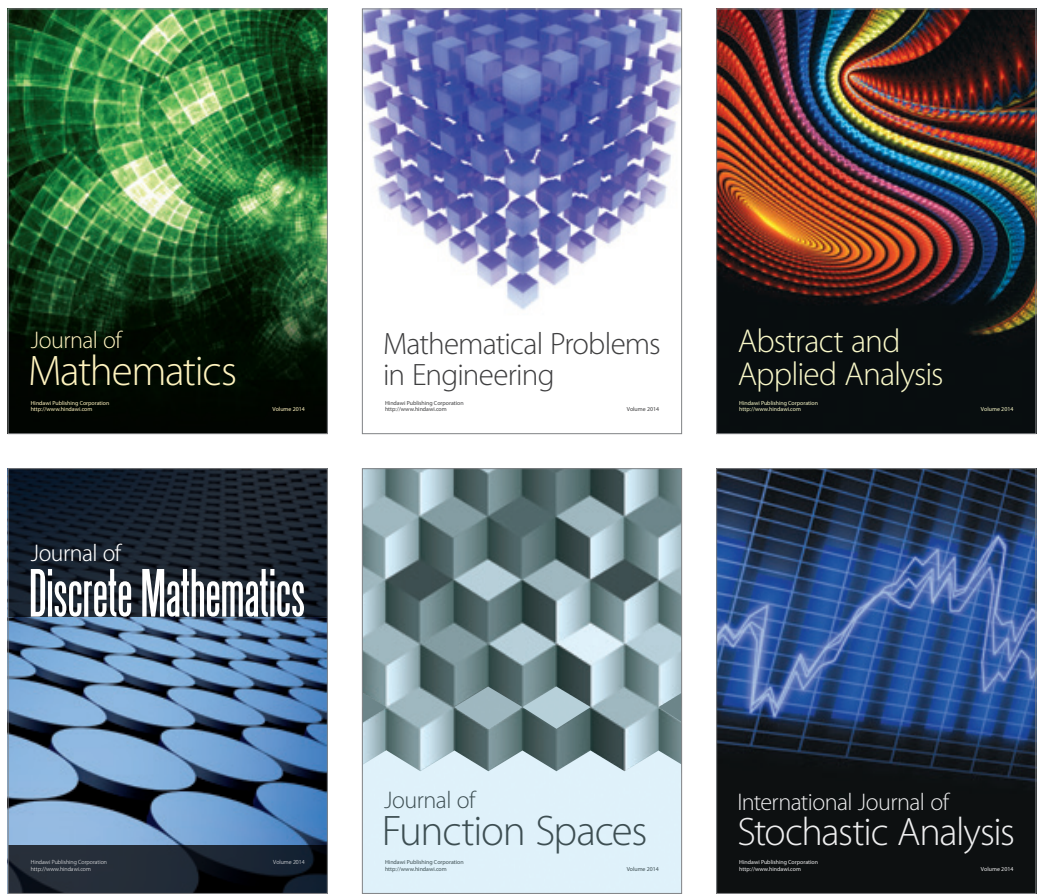

Journal of

Function Spaces

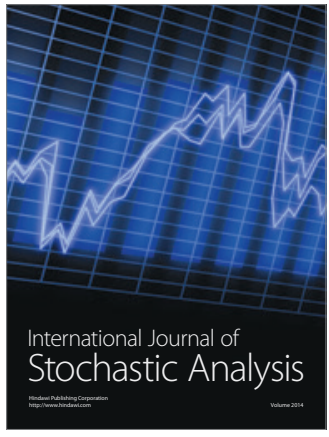

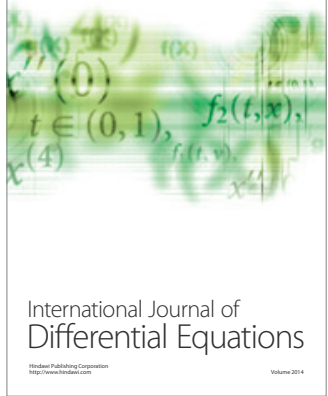
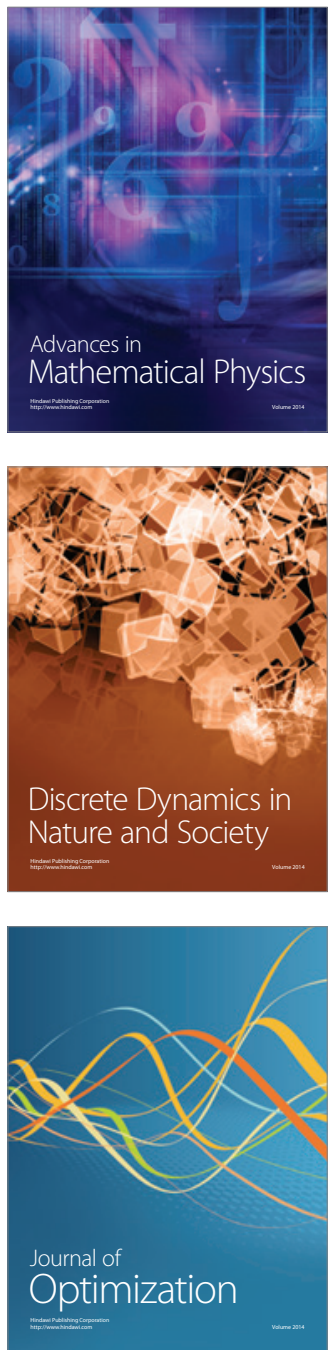